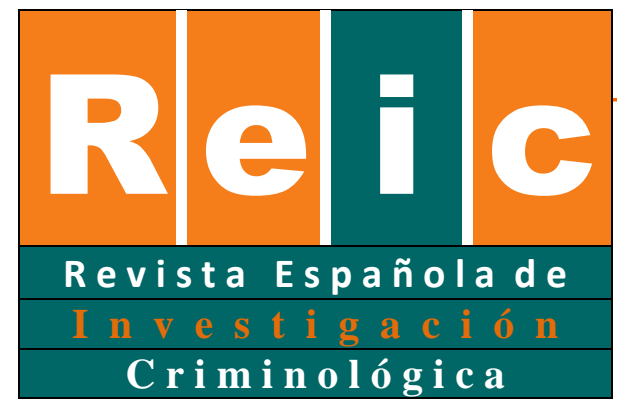

Pedrosa

\title{
¿Discrimina el Código Penal español a las mujeres?
}

\section{Is the Spanish Penal Code discriminatory against women?}

\author{
Albert Pedrosa ${ }^{1}$ iD \\ Universitat Autònoma de Barcelona
}

RESUMEN

Los datos oficiales muestran que en el contexto español se da una realidad hasta ahora poco conocida: las mujeres reciben proporcionalmente más penas de prisión que los hombres. El objetivo de este artículo es tratar de conocer si esto se debe a un fenómeno de discriminación directa, en tanto ciertos colectivos de mujeres son tratados más punitivamente; o a un fenómeno de discriminación indirecta, si el Código Penal castiga más duramente los delitos cometidos por las mujeres. Para comprobar esto se han analizado datos oficiales sobre imposición de penas y delincuencia conocida para el período 2007-15. Los resultados muestran que el alto porcentaje de penas de prisión impuestas a mujeres puede deberse a la poca libertad que otorga el Código Penal para imponer penas alternativas para delitos leves, especialmente en relación a la delincuencia patrimonial, más cometida por las mujeres, apoyando la existencia de un fenómeno de discriminación indirecta.

Palabras clave: género, penas de prisión, discriminación.

\section{ABSTRACT}

Official data shows a previously little-known reality in the Spanish context: women receive proportionally more prison sentences than men. The aim of this paper is to determine if this difference is caused by a phenomenon of direct discrimination, if judges treat certain groups of women more harshly, or by indirect discrimination, if the Penal Code is more punitive to women's offenses. Official statistics of men's and women's convictions and offenses are analyzed for the period 2007-2015. Results indicate that the higher proportion of prison sentences imposed on women could be a consequence of the

\footnotetext{
${ }^{1}$ La correspondencia debe dirigirse a: Albert Pedrosa Bou. Facultad de Derecho, Universitat Autònoma de Barcelona; C/ de la Vall Moronta, 08193 Bellaterra (Cerdanyola del Vallès), Barcelona. albert.pedrosa@uab.cat
}

Revista Española de Investigación Criminológica

Artículo 5, Número 16 (2018)

https://doi.org/10.46381/reic.v16i0.150

Www.criminologia.net

ISSN: 1696-9219 
lack of alternative sentences to prison in the Penal Code for minor offenses, especially for property crimes which are more frequently committed by women. Thus supporting the hypothesis of the existence of a phenomenon of indirect discrimination in the case of women.

Keywords: gender, prison sentences, discrimination.

\section{Introducción}

En su discurso de agradecimiento por haber recibido el premio anual otorgado por la Sociedad Europea de Criminología en 2015, y reflexionando acerca de la relación entre la criminología y la justicia, afirmaba Sonja Snacken lo siguiente: "La "Dama Justicia" generalmente es representada con los ojos vendados y sujetando una espada. Como criminólogos sabemos que haría bien en mirar un poco mejor lo que realmente está haciendo" (Snacken, 2015, pp.10). Lo cierto es que si bien la justicia puede ser ciega, los agentes del sistema penal no lo son. Contamos con evidencia de que individuos pertenecientes a determinados colectivos tienen mayor probabilidad de ser detectados y perseguidos por el sistema de justicia penal (Farrington, Loeber, \& Stouthamer-Loeber, 2003) y además, también sabemos que la decisión de imponer una pena por parte de un juez puede verse afectada por el género, la raza o la edad de la persona juzgada (Steffensmeier, Ulmer, \& Kramer, 1998).

En el contexto español existe una escasez de estudios que hayan tratado de abordar las cuestiones anteriormente descritas. Esto es especialmente preocupante en el caso del género, puesto que España se caracteriza por tener una tasa muy alta de mujeres en prisión. En este sentido, si atendemos a los datos ofrecidos por la Secretaría General de Instituciones Penitenciarias vemos cómo el porcentaje de mujeres presas en España en los últimos años ha permanecido relativamente estable entre un 7.5 y un $8 \%$, pero para afirmar que esta cifra es alta debe compararse con los países de nuestro contexto. Así, por ejemplo, los datos ofrecidos por el European Sourcebook of Crime and Criminal Justice Statistics (Aebi et al., 2014) muestran que en 2011 la tasa de mujeres encarceladas en España era de 7.6 mujeres por 100.000 habitantes; cifra que constituye la más alta de toda Europa - con la única excepción de Chipre- encontrándose muy por encima de la media 
europea, que se sitúa en 4.7. Esta situación se repite en años anteriores y posteriores, con lo que podemos afirmar que la tasa española de mujeres en prisión es una de las más altas de toda Europa ${ }^{2}$. La literatura sobre esta cuestión muestra que se produjo un gran crecimiento del encarcelamiento femenino durante los años 80 y 90, muy por encima del masculino (Almeda, 2005). Esto a su vez se ha traducido en un alto porcentaje de mujeres presas en un sistema penitenciario cuyo diseño e intervención están pensados para los hombres (Almeda, 2003; Almeda \& Bodelón, 2007; Herrera, Mapelli, \& Sordi, 2013; Maqueda Abreu, 2014; Yagüe, 2007). Sin embargo, aunque tenemos trabajos sólidos que han puesto de manifiesto esta falta de perspectiva de género en el sistema penitenciario español, contamos aún con limitada evidencia sobre cómo ha evolucionado la imposición de penas de prisión a mujeres en los últimos años.

La falta de estudios sobre la delincuencia de las mujeres y su castigo ha mantenido oculto un hecho destacable en nuestro contexto y es que, como se mostrará a continuación, los datos indican que en España, de entre el catálogo de penas disponibles, las mujeres tienden a recibir proporcionalmente más penas de prisión que los hombres. El objetivo de este trabajo es explorar si este fenómeno puede deberse o no a una discriminación hacia las mujeres por parte del sistema penal. Con esta intención se analizarán indicadores oficiales sobre imposición de penas y sobre delincuencia conocida para observar si existen diferencias por género y cómo estas pueden ser explicadas.

\section{Contextualización del fenómeno y marco teórico}

\subsection{La imposición de penas de prisión por sexo en España}

En primer lugar, y para conocer la distribución de penas por sexo, se han analizado datos oficiales ofrecidos por el Instituto Nacional de Estadística (INE) con el objetivo de ver el porcentaje que las penas de prisión ocupan dentro del total de penas impuestas para ambos sexos. La Tabla 1 muestra los resultados para el período 2007-15; se ha escogido este período debido a que los datos del INE previos a 2007 no permiten diferenciar las penas

\footnotetext{
${ }^{2}$ Los datos por año y por países se pueden consultar en las estadísticas penitenciarias europeas recogidas en las Council of Europe Annual Penal Statistics (SPACE).
} 
de prisión por sexo. Los resultados muestran que, en todos los años del período, las mujeres reciben una mayor proporción de penas de prisión. Si observamos la media de penas impuestas en dicho período para cada grupo, vemos cómo esta se sitúa en el $24 \%$ en el caso de los hombres, y en el $27.6 \%$ en el caso de las mujeres. Esto implica que las mujeres reciben casi 4 puntos porcentuales más de penas de prisión. Resulta extraño que este hecho haya merecido poca atención por parte de la academia, habiendo sido mencionado anteriormente solo por Barquín \& Luna del Castillo (2013, pp.44).

\section{Tabla 1.}

Penas de prisión impuestas por sexo sobre el total de penas en el período 2007-15

\begin{tabular}{|c|c|c|c|c|}
\hline \multirow{2}{*}{ Año } & \multicolumn{2}{|c|}{ Hombres } & \multicolumn{2}{c|}{ Mujeres } \\
\cline { 2 - 5 } & Total Penas & $\begin{array}{c}\text { Penas de prisión } \\
\text { (Total/porcentaje) }\end{array}$ & $\begin{array}{c}\text { Total } \\
\text { Penas }\end{array}$ & $\begin{array}{c}\text { Penas de prisión } \\
\text { (Total/porcentaje) }\end{array}$ \\
\hline 2007 & 408.806 & $110.131(26.9 \%)$ & 34.358 & $11.086(32.3 \%)$ \\
\hline 2008 & 582.798 & $117.915(20.2 \%)$ & 48.182 & $11.975(24, . \%)$ \\
\hline 2009 & 575.097 & $126.452(22 \%)$ & 52.655 & $13.211(25.1 \%)$ \\
\hline 2010 & 570.526 & $127.882(22.4 \%)$ & 54.073 & $13.967(25.8 \%)$ \\
\hline 2011 & 501.412 & $121.484(24.2 \%)$ & 49.975 & $14.229(28.5 \%)$ \\
\hline 2012 & 504.098 & $126.934(25.2 \%)$ & 53.695 & $15.510(28.9 \%)$ \\
\hline 2013 & 547.571 & $136.481(24.9 \%)$ & 61.330 & $17.469(28.5 \%)$ \\
\hline 2014 & 548.505 & $137.646(25.1 \%)$ & 67.135 & $19.153(28.5 \%)$ \\
\hline 2015 & 544.853 & $134.009(24.6 \%)$ & 72.843 & $18.928(26 \%)$ \\
\hline $\begin{array}{c}\text { Media } \\
\text { período }\end{array}$ & & $24 \%$ & & $27.6 \%$ \\
\hline
\end{tabular}

Fuente: Elaboración propia a partir de los datos del INE.

Si analizamos la evolución de la imposición de penas, vemos cómo las tendencias siguen lógicas similares para ambos grupos, un descenso marcado del porcentaje de penas de prisión el año 2007 seguido de un incremento de estas hasta 2011, el cual es más marcado en el caso de las mujeres, estabilizándose el porcentaje de penas de prisión a partir de ese año y hasta 2015, donde descienden de nuevo. Por otro lado, si observamos las características de las penas que se imponen, los datos muestran que la mayoría de las penas impuestas son cortas (inferiores a 2 años) significando estas, de media para el período, un $91.1 \%$ y un $92.4 \%$ de las penas que reciben hombres y mujeres respectivamente. Por otro lado, los hombres tienden a recibir un porcentaje mayor de

Revista Española de Investigación Criminológica

Artículo 5, Número 16 (2018)

WwW.criminologia.net

ISSN: 1696-9219 
penas superiores a dos y cinco años, lo cual podría corresponderse a la comisión de delitos más graves por parte de estos. Por último destaca para ambos sexos el incremento, desde 2010, de la imposición de penas cortas, el cuál es un poco más marcado para las mujeres.

Por lo tanto, los datos que aquí se han mostrado nos permiten establecer lo siguiente. En primer lugar, en España las mujeres reciben proporcionalmente más penas de prisión que los hombres. En segundo lugar, parece que esto coincide con un incremento de la imposición de penas cortas, más marcado en el caso de las mujeres. Por último, si observamos las tendencias para ambos sexos vemos que estas siguen una lógica similar. Incluso con las variaciones anuales que se producen el porcentaje de las mujeres siempre se encuentra por encima, esto hace pensar que esta diferencia en la distribución de penas de prisión por sexo no es aleatoria, sino que se debe a alguna causa subyacente en el funcionamiento del sistema penal. En este sentido, en este trabajo se analizará si este hecho puede deberse a la existencia de fenómenos de discriminación por parte del sistema penal.

\subsection{Género y discriminación}

Para analizar si el fenómeno estudiado puede deberse a procesos de discriminación en fases previas del sistema penal pueden adoptarse múltiples perspectivas. La que aquí se propone es que estos efectos discriminatorios pueden ser directos o indirectos ${ }^{3}$. Por efecto directo se entiende que ante un mismo caso las mujeres, por el solo hecho de serlo, puedan recibir un mayor trato punitivo por parte de los jueces. Esto implica que el estudio de la existencia de fenómenos de discriminación directa debe centrarse en el estadio judicial y en las variables que afectan a la imposición de penas. En cambio, cuando hablamos de discriminación indirecta, en primer lugar se debe partir de la base de que la distribución de los delitos varía en función de factores como el género. Por lo tanto, un sistema penal que no tenga en cuenta esta diferente distribución de la delincuencia puede terminar, de forma indirecta, siendo más punitivo respecto de los delitos cometidos por ciertos

\footnotetext{
3 Esta diferenciación se inspira en los conceptos de "disparate treatment" y "disparate impact" respectivamente, empleados en el derecho antidiscriminatorio estadounidense (Barrère, 2003).
} 
colectivos $^{4}$, en nuestro caso las mujeres ${ }^{5}$. Por lo tanto, en el estudio de la existencia o no de discriminación indirecta deberá analizarse la distribución de delitos cometidos por sexo, y comparar dichos datos con las penas impuestas.

En nuestro contexto contamos con poca evidencia acerca de si se dan o no estos procesos de discriminación. Esto se debe a dos hechos: En primer lugar, no se han encontrado estudios que hayan analizado el género del infractor como variable que implica mayor trato punitivo por parte de los jueces, con la única excepción de Cid \& Larrauri (2002), cuyos resultados no muestran ningún efecto significativo respecto a esta cuestión. En segundo lugar, no contamos con estudios específicos sobre delincuencia cometida por mujeres, y los estudios con muestras amplias de sujetos delincuentes, o bien no las incluyen, o bien presentan los datos sin distinción por sexo (p. ej. Díez Ripollés, 2006; García España, Díez Ripollés, Pérez Jiménez, Benítez Jiménez, \& Cerezo Domínguez, 2010), encontrando tan solo alguna excepción en estudios realizados sobre muestras de menores infractores/as (Fernández, Bartolomé, Rechea, \& Megías, 2009). Sí contamos, como se destacaba al inicio, con estudios que han puesto de manifiesto la discriminación que las mujeres pueden sufrir en prisión, pero nos queda por conocer si dicha discriminación también se produce en fases previas del proceso penal.

Todo ello implica que sea necesario recurrir a la literatura internacional sobre estos procesos de discriminación para poder establecer las bases de los conceptos que aquí se presentan:

\section{Discriminación Directa}

La cuestión clave a la hora de determinar si se da este tipo de discriminación es conocer si los agentes del sistema penal toman en consideración diferentes variables en el proceso de construcción de la categoría mujer delincuente (Hudson, 2013; Worrall, 2000) respecto al caso masculino. Los resultados de estudios específicos muestran, en líneas generales, que las mujeres reciben un trato menos punitivo, teniendo menor probabilidad de recibir

\footnotetext{
${ }^{4}$ Un ejemplo de este tipo de discriminación se puede encontrar en Blumstein (2004, pp.70) dónde se describe que la mayor punición del tráfico de crack en algunos estados de EEUU afectaba en mayor medida a la población negra, que traficaba más con dicha droga.

${ }^{5}$ Una reflexión sobre cómo las leyes pueden ejercer efectos de discriminación indirecta en base al género puede encontrarse en Larrauri (2009).
} 
una pena de prisión (Daly \& Bordt, 1995; Steffensmeier, Kramer, \& Streifel, 1993). Estos resultados parecen diferir en el caso de las infractoras jóvenes, existiendo estudios que muestran que éstas pueden recibir un mayor trato punitivo en relación a la comisión de delitos menores y delitos de estatus ${ }^{6}$ (Chesney-Lind \& Shelden 2013). A su vez, la investigación también sugiere que el trato judicial respecto a las mujeres puede variar dependiendo del tipo de delito, del perfil de la acusada, o del hecho de tener hijos a su cargo (Farnworth \& Teske, 1995; Koons-Witt, 2002; Rodriguez, Curry, \& Lee, 2006). Otro punto que debe tenerse en cuenta es la interacción del género con otras variables generadoras de discriminación. Una de las variables más destacadas por la literatura, en cuanto a su posible efecto sobre las resoluciones judiciales, es la etnia (Mitchell, 2005; Steffensmeier \& Demuth, 2001). En referencia a esto, la literatura indica que los hombres jóvenes negros o hispanos tienden a recibir un trato más punitivo (Curry \& CorralCamacho, 2008; Doerner \& Demuth, 2010; Spohn \& Holleran, 2000; Steffensmeier et al., 1998). Dicho efecto, sin embargo, parece que no se produce en el caso de las mujeres, siendo los hombres (tanto si pertenecen a minorías como sino) más duramente castigados que las mujeres con independencia del perfil de estas (Steffensmeier \& Demuth, 2006). Por lo tanto, la literatura en general no muestra que el género tenga un efecto directo sobre las condenas a mujeres. Sin embargo, la investigación sí parece indicar que esto puede cambiar respecto determinados colectivos de mujeres, con especial atención a las infractoras jóvenes y a las pertenecientes a minorías.

\section{Discriminación indirecta}

En referencia a este tipo de discriminación, resulta interesante consultar los estudios que han tratado de analizar los cambios que se han producido en las últimas décadas en las tendencias de la delincuencia de las mujeres, lo cual ha podido llevar a una modificación de la brecha de género, esto es, el hecho que los hombres cometen delitos en una proporción mucho mayor que las mujeres. Existe evidencia de que en las últimas décadas esta diferencia se ha reducido, al menos en cuanto a la delincuencia conocida (Smith, 2014), por lo que resulta importante conocer a qué puede deberse esto. Si bien la literatura

\footnotetext{
${ }^{6}$ En EEUU los delitos de estatus o status offenses son conductas que sólo son punibles si son cometidas por menores, y se refieren a "conductas problemáticas" como abuso del alcohol o huir de casa.
} 
que ha tratado de dar respuesta a esta cuestión es amplia, podríamos distinguir dos grandes líneas explicativas: la primera partiría de la base que determinados cambios sociales que se han producido en las últimas décadas, y más concretamente el acceso de la mujer al mercado laboral, su adquisición de mayores derechos, así como una mayor participación de los hombres en las tareas domésticas y de crianza, han provocado que las situaciones de ambos sexos, en cuanto a presencia de factores de riesgo y exposición a oportunidades delictivas, tiendan a acercarse, lo cual podría provocar que las mujeres cometan más delitos y/o que los hombres cometan menos. En todo caso parece que esta perspectiva ha recibido poco o limitado apoyo empírico (Steffensmeier, Zhong, Ackerman, Schwartz, \& Agha, 2006). En cambio, una segunda línea explicativa destaca que la reducción de la brecha de género es en cierto modo artificial, esto es, que las mujeres no cometen más delitos sino que los que estas cometen son más perseguidos por el sistema penal. Esto se sustenta en investigaciones que han mostrado que esta reducción de la brecha de género se produce más en algunos delitos que en otros, llevando a la conclusión de que el incremento en los últimos años de la persecución de la delincuencia violenta y la delincuencia leve patrimonial cometida por mujeres puede haber llevado a un incremento de las condenas (Estrada, Bäckman, \& Nilsson, 2015; Steffensmeier, Schwartz, Zhong, \& Ackerman, 2005). Esta última idea es especialmente interesante puesto que coincide con el concepto de discriminación indirecta que se plantea en la presente investigación.

\section{Método}

El objetivo de este artículo es dar respuesta a si la mayor imposición de penas de prisión a mujeres puede deberse a la existencia de fenómenos de discriminación directa o indirecta. Por lo tanto, se tratarán de explorar dos hipótesis relativas a cada uno de estos tipos de discriminación:

H1: Las mujeres jóvenes y extranjeras recibirán más penas de prisión (discriminación directa).

H2: Los delitos cometidos por las mujeres serán más castigados con pena de prisión (discriminación indirecta). 
Con este fin se ha optado por analizar estadísticas oficiales, en concreto aquellas ofrecidas por el Instituto Nacional de Estadística (INE), pues dichos datos nos permitirán contextualizar de forma general el fenómeno estudiado y sus posibles causas. Así pues, se analizarán los datos sobre imposición de penas de prisión y distribución de delitos ofrecidos por el INE para el período 2007-2015. Las hipótesis planteadas hacen referencia a fenómenos de discriminación que pueden tener un impacto sobre la imposición de penas en la fase judicial, por lo que parece relevante analizar las estadísticas sobre penas de prisión impuestas que publica el INE. En el análisis no se incluyen datos anteriores al año 2007 debido a que las estadísticas del INE de años anteriores no permiten el análisis de la imposición de penas de prisión por sexo (Barquín \& Luna del Castillo, 2012). Por otro lado, en el momento de realización del estudio los últimos datos disponibles eran los relativos al año 2015.

Cabe destacar que el uso de estadísticas oficiales conlleva ciertos problemas. En concreto, los datos del INE presentan dos limitaciones a tener en cuenta: en primer lugar, si bien ofrecen datos sobre condenados y penas de prisión, no incluyen datos sobre el número real de hombres y mujeres que han recibido una pena de prisión, independientemente del número de penas que reciba cada sujeto. En segundo lugar, estos datos no permiten el análisis libre de variables, por lo que el análisis debe centrarse en las categorías o combinaciones de indicadores establecidos previamente por el INE.

\section{Resultados}

\section{H1: Las mujeres jóvenes y extranjeras recibirán más penas de prisión}

Como se señalaba anteriormente, la literatura internacional sobre decisiones judiciales remarca la necesidad de analizar cómo la edad y el hecho de pertenecer a una minoría interactúan con el género. Sobre esta cuestión, los únicos datos disponibles en el INE son relativos al período 2010-15. En primer lugar, y de forma general, se han calculado los porcentajes que representan las penas de prisión impuestas a mujeres y a hombres (sobre el total de penas para cada sexo) distinguiendo entre las impuestas a personas jóvenes ${ }^{7}$,

\footnotetext{
${ }^{7}$ Se han escogido los 25 años como punto para diferenciar a los sujetos jóvenes. Se ha escogido esta edad en concreto por ser el punto a partir del cual se han encontrado mayores diferencias.
} 
así como a las personas extranjeras ${ }^{8}$. La media para el período muestra que las penas de prisión impuestas a las personas de hasta 25 años suponen un $27.6 \%$ y un $26.3 \%$ para hombres y mujeres respectivamente; por su parte, las penas impuestas a personas extranjeras suponen un $27.9 \%$ y un $26.2 \%$ respectivamente. En cuanto a la evolución de las cifras se observa un descenso sostenido (si bien leve, alrededor de un 2\%) de las penas impuestas a personas jóvenes para ambos sexos, descenso que también se da, y en mayor medida, (un $4 \%$ aproximadamente) sobre las penas impuestas a personas extranjeras. En todo caso y para todo el período, los hombres jóvenes y extranjeros reciben un mayor porcentaje de penas de prisión, aunque las cifras son parecidas respecto a las de las mujeres. Queda pues, tratar de analizar conjuntamente las anteriores variables. Los resultados de la Tabla 2 muestran que los hombres jóvenes extranjeros reciben un mayor porcentaje de penas de prisión respecto al mismo grupo de mujeres, aunque de nuevo la diferencia es mínima ( $8.3 \%$ respecto el $7.6 \%$ de media para las mujeres).

En resumen, los resultados anteriores ponen de manifiesto que la distribución de penas entre los infractores jóvenes es muy parecida en términos de género incluso si añadimos la nacionalidad como factor, lo cual no aporta indicios de que se pueda estar produciendo un fenómeno de discriminación directa en base al género. Esto es debido a que si los jueces ante un mismo caso tendieran a condenar más punitivamente (con un mayor uso de prisión) a ciertos grupos de mujeres (en nuestro caso, mujeres jóvenes y extranjeras), esto generaría que dentro del total de penas que ambos sexos reciben la pena de prisión estuviera más representada entre estos grupos de mujeres lo cual, según datos oficiales, no sucede.

\section{Tabla 2.}

Penas de prisión a mujeres y hombres extranjeros, por edad, para el período 2010-15

\begin{tabular}{|c|c|c|c|c|}
\hline \multirow{2}{*}{ Año } & \multicolumn{2}{|c|}{ Mujeres (extranjeras) } & \multicolumn{2}{c|}{ Hombres (extranjeros) } \\
\cline { 2 - 5 } & $\begin{array}{c}\text { Hasta 25 } \\
\text { años }\end{array}$ & Más de 25 años & Hasta 25 años & Más de 25 años \\
\hline 2010 & $1.219(8.7 \%)$ & $2.690(19.3 \%)$ & $11.490(9 \%)$ & $26.547(20.8 \%)$ \\
\hline 2011 & $1.249(8.8 \%)$ & $2.845(20 \%)$ & $11.241(9.3 \%)$ & $25.480(21 \%)$ \\
\hline
\end{tabular}

\footnotetext{
${ }^{8}$ Se ha optado únicamente por diferenciar entre personas con nacionalidad española y personas extranjeras por razones de espacio, aunque en el futuro sería recomendable observar si existen diferencias por distintas nacionalidades.
} 


\begin{tabular}{|c|c|c|c|c|}
\hline 2012 & $1.265(8.2 \%)$ & $2.959(19.1 \%)$ & $10.867(8.6 \%)$ & $25.071(19.8 \%)$ \\
\hline 2013 & $1.268(7.3 \%)$ & $3.140(18 \%)$ & $11.278(8.3 \%)$ & $26.542(19.4 \%)$ \\
\hline 2014 & $1.249(6.5 \%)$ & $3.390(17.7 \%)$ & $10.265(7.5 \%)$ & $25.738(18.7 \%)$ \\
\hline 2015 & $1.218(6.4 \%)$ & $3.318(17.5 \%)$ & $9.670(7.2 \%)$ & $24.416(18.2 \%)$ \\
\hline $\begin{array}{c}\text { Media } \\
\text { periodo }\end{array}$ & $7.6 \%$ & $18.6 \%$ & $8.3 \%$ & $19.6 \%$ \\
\hline
\end{tabular}

Fuente: Elaboración propia a partir de los datos del INE.

\section{H2: Los delitos cometidos por las mujeres serán más castigados con pena de prisión}

En primer lugar y debido a la falta de evidencia sobre dicha cuestión, debemos analizar si la brecha de género se ha reducido o no para el periodo estudiado. Como se puede observar en la Tabla 3, los datos coinciden con la tendencia en otros países que muestra un crecimiento sostenido de la delincuencia conocida de las mujeres, crecimiento que no es tan estable en el caso de los hombres, cuyas cifras presentan variaciones.

\section{Tabla 3.}

Delitos por 100.000 habitantes, por sexo, para el período 2007-15

\begin{tabular}{|c|c|c|}
\hline Año & Mujeres & Hombres \\
\hline 2007 & 75.8 & 888.7 \\
\hline 2008 & 94.1 & 1085.1 \\
\hline 2009 & 103.2 & 1112.7 \\
\hline 2010 & 103.7 & 1053.7 \\
\hline 2011 & 109.2 & 1076.7 \\
\hline 2012 & 117.1 & 1070.9 \\
\hline 2013 & 122.5 & 1069.4 \\
\hline 2014 & 135.5 & 1075 \\
\hline 2015 & 161.4 & 1098.7 \\
\hline
\end{tabular}

Fuente: Elaboración propia a partir de los datos del INE.

A su vez, estos datos deben acompañarse de un análisis de la distribución de los delitos para conocer si dicho incremento se ve acompañado por un aumento de la delincuencia grave de las mujeres. Esto se debe a que el mayor número de penas de prisión impuestas puede explicarse partiendo de dos supuestos diferentes: por un lado, que las mujeres cometan en proporción delitos más graves, y por lo tanto tengan más probabilidad de recibir una pena de prisión; o por otro, que el sistema de justicia sea más punitivo respecto los delitos que ellas cometen. Cabe destacar que el primer supuesto se 
corresponde con un correcto funcionamiento del sistema penal, mientras que el segundo plantea un escenario donde se está produciendo un fenómeno de discriminación indirecta como el que aquí tratamos de identificar.

Con tal fin, se ha optado por observar la distribución por sexo de diversos tipos de delitos, con especial atención a la distribución de la delincuencia grave. Se han extraído los datos sobre delincuencia del INE, y se han calculado los porcentajes sobre el total de delitos para cada sexo. La Figura 1 ilustra la distribución mediante la media para todo el período. Los datos muestran lo siguiente: en primer lugar, vemos cómo las formas de delincuencia grave se encuentran mayormente representadas entre los hombres, incluyendo el homicidio, los delitos contra la libertad sexual o las lesiones. La única excepción la encontramos en los delitos contra la salud pública, que suponen un porcentaje ligeramente superior entre las mujeres, aunque los datos del INE no permiten diferenciar por gravedad de dichas conductas. Por su parte, la delincuencia leve se encuentra más representada entre las mujeres destacando el alto porcentaje de delitos contra el patrimonio, con la única excepción de los delitos contra la seguridad vial, cuyo porcentaje es mayor entre los hombres. Estos datos parecen coincidir con la literatura previa en nuestro contexto sobre el perfil delictivo de las mujeres que llegan a prisión (Almeda, Di Nell, \& Navarro, 2012). 


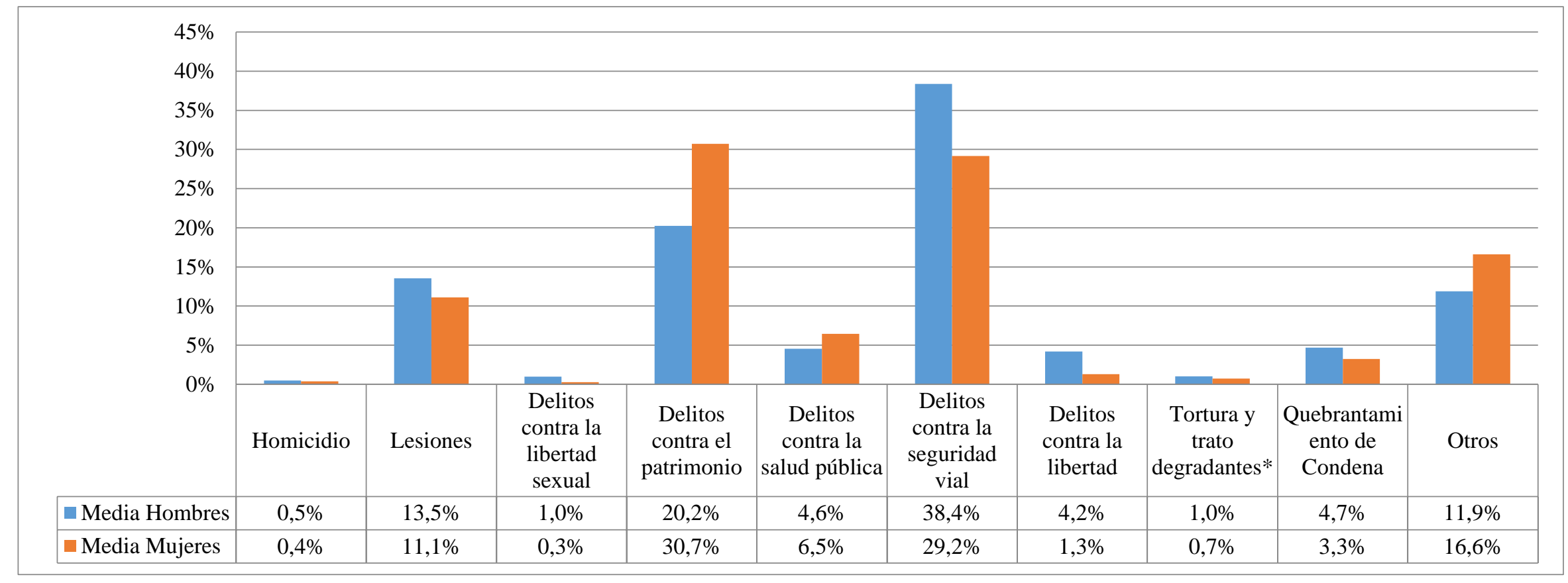

*Esta categoría incluye los supuestos de violencia habitual en el ámbito doméstico del art. 173.2 del CP Fuente: Elaboración propia a partir de los datos del INE

Figura 1. Delitos por sexo para el período 2007-15 (porcentaje medio) 
El alto porcentaje de delitos patrimoniales cometidos por mujeres justifica la necesidad de analizar los tipos penales concretos que integran dicha categoría delictiva, para distinguir los supuestos más graves como los robos de otros más leves como el hurto. La Tabla 4 muestra claramente cómo sobre el total de delitos las mujeres cometen un mayor porcentaje de hurtos, siendo este delito muy residual entre los hombres, cuyos delitos patrimoniales se centran en el robo. Sí es cierto que dentro de los delitos patrimoniales los porcentajes de hurtos y robos se encuentran menos alejados en el caso de las mujeres, pero eso se debe al número tan bajo de hurtos que cometen los hombres. Si observamos las tendencias las cifras se caracterizan por una cierta estabilidad, aunque resulta sorprendente el gran incremento del número de hurtos que se produce en 2015 para ambos sexos, siendo este muy destacable en el caso de las mujeres, donde pasan de suponer un $11.4 \%$ del total de delitos que cometen en 2014 a un $21 \%$ en 2015 . Tal y como se desarrollará posteriormente, dicho incremento puede ser consecuencia de la última reforma del Código Penal de 2015 en la que se elevan a la categoría de delitos conductas que anteriormente eran consideradas faltas de hurto, por lo que dicho incremento no estaría representando un cambio en la comisión de los delitos o su distribución, sino un cambio en el tratamiento legal de estas conductas.

\section{Tabla 4.}

Hurtos y Robos (total y porcentaje) sobre el total de delitos por sexo para el período 20072015

\begin{tabular}{|c|c|c|c|c|}
\hline \multirow{2}{*}{ Añ } & \multicolumn{2}{|c|}{ Mujeres } & \multicolumn{2}{c|}{ Hombres } \\
\cline { 2 - 5 } & Hurtos & Robos & Hurtos & Robos \\
\hline 2007 & $222(12.9 \%)$ & $1635(9.5 \%)$ & $6532(3.3 \%)$ & $24399(12.4 \%)$ \\
\hline 2008 & $2467(11.4 \%)$ & $1702(9.7 \%)$ & $6390(2.6 \%)$ & $24462(10 \%)$ \\
\hline 2009 & $2499(10.4 \%)$ & $1711(7.1 \%)$ & $5914(2.3 \%)$ & $24827(9.7 \%)$ \\
\hline 2010 & $2440(10 \%)$ & $1739(7.1 \%)$ & $6192(2.6 \%)$ & $25474(10.5 \%)$ \\
\hline 2011 & $2763(10.7 \%)$ & $1737(6.7 \%)$ & $6333(2.6 \%)$ & $26088(10.5 \%)$ \\
\hline 2012 & $3007(10.8 \%)$ & $2005(7.2 \%)$ & $6968(2.8 \%)$ & $27545(11.1 \%)$ \\
\hline 2013 & $3258(11.2 \%)$ & $2021(7 \%)$ & $7493(3 \%)$ & $28488(11.6 \%)$ \\
\hline 2014 & $3664(11.4 \%)$ & $2214(6.9 \%)$ & $7942(3.2 \%)$ & $28601(11.6 \%)$ \\
\hline 2015 & $7994(21 \%)$ & $2013(5.4 \%)$ & $12612(5 \%)$ & $27486(12 \%)$ \\
\hline $\begin{array}{c}\text { Media } \\
\text { periodo }\end{array}$ & $12.2 \%$ & $7.4 \%$ & $3 \%$ & $10.9 \%$ \\
\hline
\end{tabular}

Fuente: Elaboración propia a partir de los datos del INE. 
Por lo tanto, no sólo vemos cómo las mujeres cometen en mayor proporción delitos que pueden ser considerados leves como los patrimoniales, sino que, además, y respecto a los hombres, dentro de dicha tipología cometen en mayor proporción las conductas más leves, en este caso el hurto. Conectando esto con nuestra hipótesis, sabemos que el Código Penal Español es especialmente punitivo respecto la delincuencia leve ya que, en concreto respecto la delincuencia patrimonial, plantea la pena de prisión como la principal opción a imponer (Almeda, 2007). Como se comentará a continuación, el hecho de que el CP sea tan punitivo respecto estos delitos podría estar detrás del alto porcentaje de penas de prisión que reciben las mujeres, y a su vez podría indicar que se está produciendo un fenómeno de discriminación indirecta, puesto que el CP es más duro respecto de los delitos que más cometen las mujeres.

\section{Discusión y conclusiones}

\subsection{Discusión}

Este artículo ha tratado de dar respuesta a si el hecho de que las mujeres reciban proporcionalmente más penas de prisión se debe a un fenómeno de discriminación directa, en tanto los jueces son más punitivos respecto algunos colectivos de mujeres; o de discriminación indirecta, si el sistema penal actúa con más dureza respecto los delitos que estas cometen. De los resultados obtenidos se pueden extraer las siguientes conclusiones.

En primer lugar, y acerca de la primera hipótesis, los datos no parecen indicar que los jueces sean más punitivos respecto a determinados colectivos de mujeres. Esto es debido a que los hombres extranjeros, así como los más jóvenes, reciben penas de prisión en mayor proporción. Lo mismo sucede si observamos los resultados en base a la nacionalidad y la edad cruzadas. Por lo tanto, y aunque en el futuro sería necesario un estudio específico con variables a nivel judicial, las cifras oficiales no indican que tal fenómeno de discriminación directa pueda estar produciéndose, al menos en base al género. Esto coincide tanto con los resultados de la literatura internacional (Steffensmeier et al., 1998) como con los estudios anteriores llevados a cabo en nuestro contexto sobre dicha cuestión (Cid \& Larrauri, 2002). 
En cambio, y en referencia a la segunda hipótesis, los datos parecen indicar que sí podría estar produciéndose un fenómeno de discriminación indirecta. Los datos sobre distribución de los delitos nos muestran cómo las mujeres cometen un mayor porcentaje de delitos leves respecto a los hombres. Esto es consistente con la literatura internacional sobre la delincuencia cometida por mujeres (Chesney-Lind \& Pasko, 2012; Steffensmeier \& Allan, 1996; Walklate, 2013). En concreto, destaca la mayor comisión por parte de estas de delitos contra el patrimonio, mayoritariamente hurtos. Esto es significativo puesto que el CP español no permite la aplicación como pena principal de penas alternativas a la prisión para este tipo de delitos, lo cual implica que las personas que cometan más estas conductas tendrán más probabilidad de recibir una pena de prisión. Por lógica, entonces, una proporción tan alta de delitos contra el patrimonio debería suponer inevitablemente un alto número de penas de prisión.

Existe, sin embargo, una excepción a esto, y es que los hombres cometen en una mayor proporción delitos contra la seguridad vial, delitos que también incluyen muchas conductas leves como la conducción sin permiso. Este caso pone en cuestión los argumentos que se han planteado anteriormente, puesto que, si el sistema penal es expansivo en cuanto a una mayor punitividad respecto de los delitos leves, los hombres deberían también recibir un gran número de penas de prisión. Esto parece no ser así y la razón, de acuerdo con los resultados que se han mostrado en este artículo, se encuentra nuevamente en un diseño del CP que carece de perspectiva de género. Si analizamos los cambios legislativos en materia de seguridad vial, podemos comprobar cómo las reformas de 2003 y 2007 dirigidas a transformar en delito conductas que anteriormente eran constitutivas de falta o infracción administrativa produjeron un incremento de las condenas por este tipo de supuestos. Sin embargo, el legislador fue previsor y optó por permitir que los jueces pudieran imponer como pena principal alternativas a la prisión como la multa o los trabajos en beneficio de la comunidad para responder a unas conductas delictivas que se entendían como leves ${ }^{9}$. Esto, que supone una buena praxis por parte del sistema penal -que se puedan imponer como pena principal alternativas a la prisión para delitos leves- sin embargo, ha terminado afectando tan solo a

\footnotetext{
${ }^{9}$ De hecho, el gran aumento en la imposición de penas de TBC producido a raíz de estas reformas refuerza este argumento (Blay, 2010).
} 
algunas de estas conductas leves, y casualmente -o no- a las más cometidas por los hombres. Lo que aquí planteo es que el CP y sus intervenciones se han diseñado pensando en un sujeto delincuente cuya categoría se corresponde con la de hombre delincuente, por lo que en la práctica se produce un olvido de las mujeres infractoras, sus características y necesidades específicas, olvido que es una nueva manifestación de discriminación indirecta. Por lo tanto, el factor diferencial entre las penas impuestas por sexo no es la mayor punición de la delincuencia leve, sino un trato más punitivo respecto a determinada delincuencia leve (patrimonial) que es más cometida por las mujeres ${ }^{10}$.

En la misma línea llama la atención el gran incremento de los hurtos cometidos en 2015, especialmente significativo en el caso de las mujeres, puesto que supone un incremento de casi un $10 \%$ respecto 2014. Esto se debe a que la reforma del CP de marzo de 2015 elevó a delito los supuestos considerados anteriormente como faltas (hurto de cantidades inferiores a 400 euros), lo cual ha supuesto un lógico incremento de estos delitos. Sin embargo esto no se ha traducido en un aumento de las condenas de prisión de las mujeres como sería esperable según la tesis que aquí se ha defendido. La razón se encuentra en que la nueva modificación del art. 234.2 del CP ya establece que los supuestos donde la cantidad hurtada sea menor de 400 euros (lo que anteriormente eran faltas) se castiguen con pena de multa, y no privativa de libertad, como sí se castiga el supuesto clásico de hurto. Por lo tanto, nuevamente esto pone de manifiesto que cuando el $\mathrm{CP}$ prevé la imposición de penas alternativas, esto obviamente evita un incremento de las penas de prisión impuestas para supuestos leves.

A modo de resumen, todo lo anterior implica que las mujeres no reciben más penas de prisión porque cometan delitos más graves, sino porque cometen determinadas formas de delincuencia leve que son castigadas con mayor severidad. A su vez, esto podría explicar la reducción de la brecha de género en los últimos años, la cual se ha desarrollado en paralelo

\footnotetext{
${ }^{10}$ En contraposición a esto podría argumentarse que los hombres al cometer muchos delitos que implican penas accesorias (p. ej. retirada del permiso de conducir) ven incrementado el total de penas que reciben, reduciendo el porcentaje que representan las penas de prisión. Sin embargo y aunque no se han podido incluir aquí por razones de espacio, se han realizado los mismos análisis sobre el total de penas de imposición directa, obteniendo los mismos resultados, por lo que la pregunta relevante sigue siendo la misma, por qué las mujeres, cometiendo delitos más leves, en proporción son más castigadas con prisión. Agradezco al prof. Daniel Varona el comentario sobre dicha cuestión.
} 
al incremento de los delitos patrimoniales y de las penas cortas. Aunque se requiere de un análisis específico para demostrar esto, esta hipótesis sobre los efectos de discriminación indirecta tiene coherencia y parece coincidir con lo que otros autores han puesto de manifiesto en otros contextos (Estrada, Bäckman, \& Nilsson, 2015), donde se ha observado que el incremento de la acción penal hacia conductas cada vez más leves ha afectado en mayor medida a las mujeres.

Llegados a este punto un último tema clave que queda por conocer es si en el fondo, y siguiendo los postulados básicos de la Criminología Crítica, esta dinámica expansiva del sistema penal afecta de manera discriminatoria a las mujeres como colectivo, o si por el contrario, lo hace sobre las personas de las clases desfavorecidas con independencia de su sexo. No se trata de establecer una jerarquía en relación a la discriminación, sino de reconocer la interseccionalidad de esta, esto es, conocer si en realidad el sistema de justicia y el CP están siendo más punitivos respecto a las personas con situaciones de mayor desventaja estructural, situación que se ve acrecentada en el caso de las mujeres al ser objeto estas de otros procesos de discriminación. El problema no radica en que algunos delitos sean más castigados que otros, sino en el hecho que lo sean los cometidos en mayor medida por determinados colectivos, siendo especialmente grave si se trata de delitos leves. Por lo tanto, la última cuestión que se debe plantear es si esta discriminación indirecta afecta a todas las mujeres, o si esta afecta más a las mujeres de las clases desfavorecidas. En relación con esto, no debe olvidarse que si bien las mujeres gitanas son un colectivo representativo en prisión (Naredo, 2005), estas permanecen invisibles ante las estadísticas oficiales, puesto que estas solo contienen datos relativos a la nacionalidad. Para abordar dicha cuestión, en el futuro se debería contar con datos relativos a la situación socioeconómica de las personas condenadas a prisión.

\subsection{Conclusiones e implicaciones}

La preocupación inicial que daba lugar a este artículo era la siguiente: los datos indican que en España las mujeres reciben proporcionalmente más penas de prisión que los hombres. Desafortunadamente, los datos disponibles y la amplitud del objeto de estudio no han 
permitido más que aportar unas primeras conclusiones generales y puntos básicos que permitan guiar la investigación futura sobre esta cuestión. Sin embargo, la generalidad de los resultados no debe restar importancia a algunos de los fenómenos que aquí se han puesto de manifiesto, en especial al hecho de que esta mayor proporción de penas de prisión puede deberse a la mayor punición de los delitos leves, los cuales a su vez son más cometidos por las mujeres. Esto lleva a plantear que puede estar produciéndose un fenómeno de discriminación indirecta sobre las mujeres delincuentes, consecuencia de la falta de perspectiva de género en el código penal.

Queda, pues, en manos de la investigación futura tratar de abordar las cuestiones que aquí se han planteado empleando una mayor amplitud de indicadores puesto que los datos oficiales, debido a sus limitaciones, no parecen ser los más adecuados para abordar algunos de los puntos clave que se han introducido como el efecto del género en las decisiones judiciales o conocer si la reducción de la brecha de género se debe a un aumento de los delitos cometidos por mujeres o por el contrario a una mayor persecución de esta, entre otros. Para ello resultaría imprescindible contar, por un lado, con estudios específicos sobre mujeres delincuentes, y por otro, con estudios que sigan las trayectorias de estas a lo largo de las distintas fases del sistema penal. Sólo así podríamos abordar otra de las cuestiones que parecen surgir de los resultados presentados, conocer si esta discriminación indirecta afecta más a las mujeres pertenecientes a minorías o clases más desfavorecidas.

No se trata en ningún caso de proponer que exista un $\mathrm{CP}$ para cada género, sino de que el CP común se diseñe teniendo en cuenta también lo que sabemos (y todo lo que nos queda por conocer) sobre la delincuencia de las mujeres. Los datos muestran que el género tiene un efecto sobre la distribución de los delitos, y esto implica que también debemos tener en cuenta el efecto del género a la hora de diseñar los castigos. Nueva evidencia es necesaria en el futuro, con el objetivo de suscitar el debate académico e institucional en pro de un CP con visión de género cuyo diseño de los castigos responda a las características y necesidades de las mujeres infractoras; solo así podremos, aunque sea en parte, avanzar hacia un sistema penal más justo. 


\section{Referencias}

Aebi, M. F., Aubusson de Cavarlay, B., Barclay, G., Gruszczyńska, B., Harrendorf, S., Heiskanen, M., ... \& Pórisdóttir, R. (2014). European sourcebook of crime and criminal justice statistics-2014. Boom Juridische Uitgevers.

Almeda, E. (2003). Mujeres encarceladas. Barcelona: Ariel.

Almeda, E. (2005). Women's imprisonment in Spain. Punishment \& Society, 7(2), 183-199.

Almeda, E. (2007). Ejecución penal y mujer en España: Olvido, castigo y domesticidad. Mujeres y castigo: Un enfoque socio-jurídico y de género, 27-66.

Almeda, E., \& Bodelón, E. (2007). Mujeres y castigo: Un enfoque socio-jurídico y de género. Madrid: Dykinson.

Almeda, E., Di Nell, D., \& Navarro, C. (2012). Mujeres, cárceles y drogas: datos y reflexiones (Women, Prisons and Drugs: Data and Reflections). Oñati Socio-Legal Series, 2(6), 122-145.

Barquín, J. S., \& Luna del Castillo, J. D. (2012). En los dominios de la prisión: Distribución numérica de las penas en el Código y en la justicia penal. Revista electrónica de ciencia penal y criminología, 16(14), 1-52.

Barquín, J. S., \& Luna del Castillo, J. D. (2013). Aplicación práctica de la suspensión y la sustitución de las penas privativas de libertad: una aproximación estadística. Revista de derecho penal y criminología, 10(3), 415-470.

Barrère, M. U. (2003). Problemas del derecho antidiscriminatorio: subordinación versus discriminación y acción positiva versus igualdad de oportunidades. Cuadernos electrónicos de filosofía del derecho, (9), 4-26.

Blay, E. (2010). 'It Could Be Us': Recent Transformations in the Use of Community Service as a Punishment in Spain. European Journal of Probation, 2(1), 62-81.

Blumstein, A. (2004). Restoring Rationality in Punishment Policy. En M. Tonry (Ed), The future of imprisonment (pp. 61-82). Oxford, London: Oxford University Press.

Chesney-Lind, M., \& Pasko, L. (2012). The female offender: Girls, women, and crime. California: Sage.

Chesney-Lind, M., \& Shelden, R. G. (2013). Girls, delinquency, and juvenile justice. Chichester: John Wiley \& Sons.

Cid, J. (Coord.), Larrauri, E. (Coord.), Escobar, G., Lahoz, J., López i Ferrer, M., Tébar, B., \& Varona, D. (2002). Jueces penales y penas en España. Aplicación de las penas alternativas a la privación de libertad. Valencia: Tirant lo blanch.

Curry, T. R., \& Corral-Camacho, G. (2008). Sentencing young minority males for drug offenses Testing for conditional effects between race/ethnicity, gender and age during the US war on drugs. Punishment \& Society, 10(3), 253-276.

Daly, K., \& Bordt, R. L. (1995). Sex Effects and Sentencing: An Analysis of the Statistical Literature. Justice Quarterly, 12(1), 141-175. DOI: 10.1080/07418829500092601

Díez Ripollés, J. L. (2006). Algunos rasgos de la delincuencia en España a comienzos del siglo XXI. Revista Española de Investigación Criminológica, (4), 1-19.

Doerner, J. K., \& Demuth, S. (2010). The independent and joint effects of race/ethnicity, gender, and age on sentencing outcomes in US federal courts. Justice Quarterly, 27(1), 1-27. DOI: 10.1080/07418820902926197

Revista Española de Investigación Criminológica

Artículo 5, Número 16 (2018)

www.criminologia.net

ISSN: 1696-9219 
Estrada, F., Bäckman, O., \& Nilsson, A. (2015). The Darker Side of Equality? The Declining Gender Gap in Crime: Historical Trends and an Enhanced Analysis of Staggered Birth Cohorts. British Journal of Criminology, 56(6), 1272-1290. DOI: 10.1093/bjc/azv114

Farnworth, M., \& Teske, R. H. (1995). Gender differences in felony court processing: Three hypotheses of disparity. Women \& Criminal Justice, 6(2), 23-44. DOI: 10.1300/J012v06n02_02

Farrington, D. P., Loeber, R., \& Stouthamer-Loeber, M. (2003). How can the relationship between race and violence be explained? En D. F. Hawkins, Violent crimes: Assessing race and ethnic differences (pp.213-237). Cambridge: Cambridge University Press.

Fernández, E., Bartolomé, R., Rechea, C., \& Megías, A. (2009). Evolución y tendencias de la delincuencia juvenil en España. Revista Española de Investigación Criminológica, 8(7), 1-30.

García España, E., Díez Ripollés, J. L., Pérez Jiménez, F., Benítez Jiménez, M. J., \& Cerezo Domínguez, A. I. (2010). Evolución de la delincuencia en España: Análisis longitudinal con encuestas de victimización. Revista Española de Investigación Criminológica, (8), 1-27.

Herrera, M. M., Mapelli, C. B., \& Sordi, B. S. (2013). La exclusión de las excluidas. ¿Atiende el sistema penitenciario a las necesidades de género?: una visión andaluza. Estudios penales y criminológicos, (33), 59-95.

Hudson, B. (2013). Gender Issues in penal policy and penal theory. En P. Carlen (Ed.), Women and Punishment: The Struggle for Justice. Cullumpton: Willan.

INE. (Varios años). Estadística de condenados. Madrid: Instituto Nacional de Estadística.

Koons-Witt, B. A. (2002). The effect of gender on the decision to incarcerate before and after the introduction of sentencing guidelines. Criminology, 40(2), 297-328. DOI: 10.1111/j.1745-9125.2002.tb00958.x

Larrauri, E. (2009). Desigualdades sonoras, silenciosas y olvidadas: género y derecho penal. Anuario de la Facultad de Derecho de la UAM, 13, 37-55.

Maqueda Abreu, M. L. (2014). Razones y sinrazones para una criminología feminista. Madrid: Dykinson.

Mitchell, O. (2005). A meta-analysis of race and sentencing research: Explaining the inconsistencies. Journal of Quantitative Criminology, 21(4), 439-466.

Naredo, M. (2005). ¿Qué nos enseñan las reclusas? La criminalización de la pobreza desde la situación de reclusas extranjeras y gitanas. Universidad de León; Humanismo y trabajo social, 67-94

Rodriguez, F. S., Curry, T. R., \& Lee, G. (2006). Gender Differences in Criminal Sentencing: Do Effects Vary Across Violent, Property, and Drug Offenses? Social Science Quarterly, 87(2), 318-339.

Smith, G. (2014). Long-Term Trends in Female and Male Involvement in Crime. En R. Gartner, \& B. McCarhty (Eds), The Oxford Handbook of Gender, Sex, and Crime (pp.139-157). Oxford, London: Oxford University Press.

Snacken, S. (2015). Criminology between science and justice. ESC Criminology in Europe: Newsletter of the European Society of Criminology, 14(3), 9-13.

Spohn, C., \& Holleran, D. (2000). The imprisonment penalty paid by young, unemployed black and Hispanic male offenders. Criminology, 38(1), 281-306.

Revista Española de Investigación Criminológica

Artículo 5, Número 16 (2018)

https://doi.org/10.46381/reic.v16i0.150

www.criminologia.net

ISSN: 1696-9219 
Steffensmeier, D., \& Allan, E. (1996). Gender and crime: Toward a gendered theory of female offending. Annual review of sociology, 22, 459-487.

Steffensmeier, D., \& Demuth, S. (2001). Ethnicity and judges' sentencing decisions: Hispanic-black-white comparisons. Criminology, 39(1), 145-178. DOI: 10.1111/j.1745-9125.2001.tb00919.x

Steffensmeier, D., \& Demuth, S. (2006). Does gender modify the effects of race-ethnicity on criminal sanctioning? Sentences for male and female white, black, and Hispanic defendants. Journal of Quantitative Criminology, 22(3), 241-261.

Steffensmeier, D., Kramer, J. H., \& Streifel, C. (1993). Gender and Imprisonment Decisions. Criminology, 31, 441-446. DOI: 10.1111/j.1745-9125.1993.tb01136.x

Steffensmeier, D., Schwartz, J., Zhong, H., \& Ackerman, J. (2005). An assessment of recent trends in girls' violence using diverse longitudinal sources: is the gender gap closing? Criminology, 43(2), 355-406. DOI: 10.1111/j.0011-1348.2005.00011.x

Steffensmeier, D., Ulmer, J., \& Kramer, J. (1998). The interaction of race, gender, and age in criminal sentencing: The punishment cost of being young, black, and male. Criminology, 36(4), 763-798. DOI: 10.1111/j.1745-9125.1998.tb01265.x

Steffensmeier, D., Zhong, H., Ackerman, J., Schwartz, J., \& Agha, S. (2006). Gender gap trends for violent crimes, 1980 to 2003 A UCR-NCVS comparison. Feminist Criminology, 1(1), 72-98.

Walklate, S. L. (2013). Gender, crime and criminal justice. Cullumpton: Willan.

Worrall, A. (2000). Governing Bad Girls: Changing Constructions of Female Juvenile Delinquency. En J. Bridgeman, \& D. Monk (Eds.), Feminist Perspectives on Child Delinquency Law. London: Cavendish Publishing.

Yagüe, C. O. (2007). Mujeres en prisión. Intervención basada en sus características, necesidades y demandas. Revista Española de Investigación Criminológica, (5), 124.

\section{Agradecimientos}

Quiero agradecer la ayuda y los consejos aportados por la Prof. Ester Blay en versiones anteriores de este artículo, así como muy especialmente al Prof. José Cid en las versiones más recientes de este. También quiero agradecer el esfuerzo y los interesantes comentarios de amigos y compañeros que han ayudado a mejorarlo. Este proyecto no cuenta con financiación de ningún organismo público o privado.

Albert Pedrosa Bou es graduado en Criminología por la Universitat Autònoma de Barcelona y Máster en Criminología y Ejecución Penal por la Universitat Pompeu Fabra. Actualmente se encuentra vinculado al grupo de investigación "Desistimiento del Delito y Políticas de Reinserción" de la Universitat Autònoma de Barcelona como investigador predoctoral (Beca FPI 2015). Sus temas de interés son el desistimiento delictivo, la delincuencia y el control penal sobre las mujeres, y el estudio del uso de la prisión.

(iD https://orcid.org/0000-0002-3533-7282

Revista Española de Investigación Criminológica

Artículo 5, Número 16 (2018)

https://doi.org/10.46381/reic.v16i0.150

Www.criminologia.net

ISSN: 1696-9219 